\title{
Extent of vitamin A deficiency among rural pregnant women in Bangladesh
}

\author{
Vanessa Lee ${ }^{1}$, Faruk Ahmed ${ }^{2,3, *}$, Shoko Wada ${ }^{4}$, Tahmeed Ahmed ${ }^{5,6}$, \\ AM Shamsir Ahmed ${ }^{5}$, Cadi Parvin Banu ${ }^{7}$ and Nasima Akhter ${ }^{8}$ \\ ${ }^{1}$ School of Public Health, Griffith University, Brisbane, Australia: ${ }^{2}$ Department of Family Sciences, College for \\ Women, Kuwait University, Safat 13060, Kuwait: ${ }^{3}$ School of Population Health, The University of Queensland, \\ Queensland, Australia: ${ }^{4}$ Japan International Cooperation Agency, Tokyo, Japan: ${ }^{5}$ International Centre for \\ Diarrhoeal Disease, Mohakhali, Dhaka, Bangladesh: ${ }^{6} J a m e s$ P Grant School of Public Health, BRAC University, \\ Dhaka, Bangladesh: ${ }^{7}$ Institute of Nutrition and Food Science, University of Dhaka, Dhaka, Bangladesh: ${ }^{8} \mathrm{Helen}$ \\ Keller International, Dhaka, Bangladesh
}

Submitted 11 October 2007: Accepted 16 April 2008: First published online 12 June 2008

\begin{abstract}
Objective: To investigate the prevalence of vitamin A deficiency (VAD) among pregnant women in rural Bangladesh, and examine the relationship between various factors and vitamin A status.

Setting: Community Nutrition Promoter (CNP) centres in Kapasia sub-district of Gazipur district, Bangladesh.

Design: A cross-sectional study.

Subjects and methods: Two hundred women, aged 18-39 years, in their second or third trimester of pregnancy were selected from seventeen CNP centres in four unions of Kapasia sub-district where they usually visit for antenatal care. Various socio-economic, personal and pregnancy-related information, dietary intake of vitamin A and mid-upper arm circumference (MUAC) data were collected. Serum retinol (vitamin A) concentration was determined.

Results: More than half ( $51 \%$ ) of the pregnant women had low vitamin A status (serum retinol $<1.05 \mu \mathrm{mol} / \mathrm{l}$ ) with $18.5 \%$ having VAD (serum retinol $<0.70 \mu \mathrm{mol} / \mathrm{l})$. Fifty-three per cent of the women's vitamin A intake was less than the recommended dietary allowance. By multiple regression analysis, MUAC, per-capita expenditure on food and wealth index were found to have significant independent positive relationship with serum retinol concentration, while gestational age of the pregnant women had a negative relationship. The overall $F$-ratio $(10 \cdot 3)$ was highly significant $(P=0 \cdot 0001)$, the adjusted $R^{2}$ was $0 \cdot 18$ (multiple $R=0 \cdot 45$ ).

Conclusion: VAD is highly prevalent among rural pregnant women in Bangladesh. Gestational age, nutritional status, per-capita expenditure on food and wealth index appear to be important in influencing the vitamin A status of these women. An appropriate intervention is warranted in order to improve the vitamin A status.
\end{abstract}

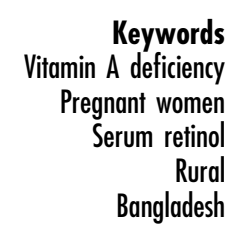

Vitamin A deficiency (VAD) is known to be a significant public health problem around the world and it is particularly serious in Africa and South-East Asia including Bangladesh ${ }^{(1)}$. Furthermore, it is increasingly recognised that pregnant and lactating women in lowincome countries are also one of the most vulnerable groups $^{(2,3)}$. Studies have shown that pregnant women with VAD are at increased risk of night blindness ${ }^{(3,4)}$ and VAD in pregnancy is also associated with anaemia ${ }^{(5)}$. It has recently become evident that VAD has important consequences on the morbidity and mortality of pregnant women ${ }^{(6,7)}$ and it may affect the pregnancy outcomes $^{(4,8)}$.
The primary cause of VAD is inadequate intake of vitamin A. Underlying causes include social-cultural and social-economical factors such as poverty, limited access to vitamin A-rich foods, low education and cultural beliefs and dietary practices ${ }^{(9,10)}$.

In Bangladesh, $\mathrm{VAD}$ has long been recognised as a major public health problem ${ }^{(11,12)}$. Over the past few decades, several studies including national nutritional surveys have been conducted to investigate the prevalence of VAD among different population groups in Bangladesh. However, most of the studies emphasised pre-school children, and therefore the data on pregnant women, especially using biochemical methods to assess VAD, are 
limited. The most recent national vitamin A survey in 1997-98 revealed that nearly one quarter of pregnant women in rural Bangladesh had VAD and only $2 \cdot 7 \%$ were suffering from night blindness, a sign of severe $\mathrm{VAD}^{(12)}$. However, this survey was not designed systematically to characterise the extent of VAD in pregnant women in rural Bangladesh and thus the report was based on a small sample size. Furthermore, since 1998, no survey to assess VAD among rural pregnant women has been carried out. Considering the importance of VAD, the present study was designed to determine the prevalence of VAD among rural pregnant women in Bangladesh. We also examined the relationship between vitamin A status and various sociodemographic, dietary and nutritional factors.

\section{Subjects and methods}

\section{Subject identification and selection}

The study was conducted in Kapasia sub-district of Gazipur district, situated in North of Dhaka, Bangladesh, which consists of eleven unions with 231 villages and 77030 households. In Kapasia, about 3500 women were reported pregnant in the year of this survey ${ }^{(13)}$. Kapasia has access to better family planning support through the non-government organisation. It has safe drinking water and good sanitation facilities, and the economic activities in the area include mostly farming and small trading.

During February-March 2006, a cohort of 200 women, aged 18-39 years, in their second or third trimester of pregnancy from seventeen Community Nutrition Promoter (CNP) centres in four unions (Sador, Turguo, Dargapur and Barishabo) in Kapasia, participated in the study. The sample for the present study was calculated on the basis of the prevalence $(23.7 \%)$ of VAD (serum retinol concentration $<0.70 \mu \mathrm{mol} / \mathrm{l}$ ) among pregnant women in the previous study in 1997 by Helen Keller International (HKI) ${ }^{(12)}$, and with an estimated $95 \%$ confidence interval of $17 \cdot 8,29 \cdot 6 \%$.

The purpose and procedures of the study were explained to the participants and they were encouraged to give their consent in writing, before being accepted into the study. The study protocol was approved by the Ethical Review Committees of the Bangladesh Medical Research Committee, Dhaka; Bangladesh Ministry of Health and Family Welfare, Dhaka; National Nutrition Programme, Dhaka, Bangladesh; and the University of Queensland, Brisbane, Australia.

\section{Questionnaire and sample collection}

A structured questionnaire was developed and pre-tested to obtain sociodemographic, personal and pregnancyrelated information of the participants. Length of gestation was calculated from the last menstrual period and expected date of delivery, and confirmed from the CNP centre's record. The wealth index, an indicator of socio-economic status, was constructed using principal components analysis. Wealth included in the index ranges from the possession of durable goods ${ }^{(14)}$. The principal components analysis of household wealth retained one factor and assigned a factor score to each household. The higher the score, the greater the number of household assets. The estimation of expenditure on food was based on the food items that were purchased from the market per week. Thus the cost of home-grown food they consumed was not considered. A $24 \mathrm{~h}$ vitamin A semiquantitative (VASQ) method was used to assess dietary vitamin A intake. The VASQ method was developed and validated $^{(15)}$, and has been widely used in nutritional surveys throughout Indonesia, Bangladesh and Nepal ${ }^{(16)}$. Briefly, a $24 \mathrm{~h}$ recall questionnaire was applied that included all foods and drinks consumed during the previous day. All vitamin A-containing ingredients were then assigned a food code and vitamin A-containing code. Vitamin A values were predominantly taken from the food composition table developed by $\mathrm{HKI}^{(17)}$. The food codes defined whether the ingredients were a vegetable, fruit, animal food or fortified food. Vitamin A content codes were assigned for the amount of vitamin A in the individual ingredient consumed, and were classified as $<20,20-75,76-150,151-300,301-750$ and $>750$ retinol activity equivalent (RAE). Vitamin A intake was calculated per food codes using the mid-points of the vitamin A content categories $^{(16)}$.

Five millilitres of blood was drawn from the subject's arm. Interviews were then carried out for the structured questionnaires and the $24 \mathrm{~h}$ recall VASQ method, and then the mid-upper arm circumference (MUAC) was measured. The blood was placed in a glass centrifuge tube and immediately wrapped in foil to protect against degradation of vitamin A by light. After centrifugation, serum samples were separated and kept frozen at $-20^{\circ} \mathrm{C}$ until further analysis. Serum retinol (vitamin A) analysis was carried out within a month of blood collection.

\section{Antbropometry and biochemical measurement}

The MUAC was measured for each participant at the mid-point of the upper left arm between the shoulder and elbow using a measuring tape to the nearest $1.0 \mathrm{~mm}$. Maternal MUAC is a potential indicator of maternal nutritional status and is related to birth outcome. Undernutrition was defined by MUAC $<22.5 \mathrm{~cm}$ since this cut-off was used to predict neonatal morbidity among the Bangladeshi population $^{(18)}$. Serum retinol was determined by HPLC according to Bieri et al. ${ }^{(19)}$ with modifications ${ }^{(20)}$. Coefficient of variations for retinol assay was determined using the pooled serum sample. The intra-assay was $3 \cdot 1 \%$ using seven replicates.

\section{Statistical analysis}

Data were analysed with the SPSS/version 12.0 (SPSS Inc., Chicago, IL, USA). 
Univariate analysis comprised a simple frequency distribution of selected variables. For MUAC, serum retinol and dietary intake of vitamin A, normality test for distribution of data was performed by the KolmogorovSmirnov goodness of fit test. The data were divided into groups on the basis of a priori logical categories for various factors. The means and differences between groups were assessed using one-way ANOVA. Pearson's correlation test was performed to examine the association between serum retinol and various social, personal and nutritional factors. Backward stepwise multiple regression analysis was carried out to examine the independent relationship of serum retinol with selected variables. The final regression model showed the ordinary least squares regression coefficients $(B)$, standard error of $B(\operatorname{se} B)$ and standardised Beta coefficients $(\beta), \beta$ is adjusted for $\mathrm{SD}$

Table 1 Characteristics of the study participants*

\begin{tabular}{|c|c|}
\hline Variable & Frequency (\%) \\
\hline \multicolumn{2}{|l|}{ Age group (years) } \\
\hline $18-19$ & $21 \cdot 5$ \\
\hline $20-29$ & $65 \cdot 0$ \\
\hline $30-39$ & $13 \cdot 5$ \\
\hline \multicolumn{2}{|l|}{ Gestational age (weeks) } \\
\hline 13-24 (second trimester) & $37 \cdot 5$ \\
\hline$>25$ (third trimester) & $62 \cdot 5$ \\
\hline \multicolumn{2}{|l|}{ Education level } \\
\hline Illiterate/informal education & $14 \cdot 0$ \\
\hline Primary & $21 \cdot 0$ \\
\hline Secondary and above & $65 \cdot 0$ \\
\hline \multicolumn{2}{|l|}{ Participant's occupation } \\
\hline Housewives & $88 \cdot 5$ \\
\hline Others & 11.5 \\
\hline \multicolumn{2}{|l|}{ Husband's occupation } \\
\hline Daily labour/farmer & $53 \cdot 5$ \\
\hline Service/business & $46 \cdot 5$ \\
\hline \multicolumn{2}{|l|}{ Family size } \\
\hline Small, $\leq 4$ members & $56 \cdot 5$ \\
\hline Medium or large, $\geq 5$ members & $43 \cdot 5$ \\
\hline \multicolumn{2}{|c|}{ Per-capita monthly incomet (Taka $\neq$ /month) } \\
\hline$<659$ \$ & $10 \cdot 5$ \\
\hline$\geq 659$ & $89 \cdot 5$ \\
\hline
\end{tabular}

${ }^{*} n 200$.

tIncome was missing for ten subjects.

$\ddagger$ Taka $68 \cdot 5=$ US $\$ 1 \cdot 0$.

§Poverty line ${ }^{(21)}$. and, being in the same unit, is directly comparable. The multiple $R^{2}$ is a measure of the goodness of fit of the regression model and the adjusted $R^{2}$ is a modification $R^{2}$ that adjusts for the number of independent variables in a model. A significance level of 0.05 was used for all statistical tests, and two-tailed tests were used.

\section{Results}

Almost two-thirds (65\%) of the participants were aged between 20 and 29 years (Table 1). Only $14 \%$ of the women were illiterate or had informal education. Nearly $89 \%$ of the participants were housewives. More than half $(53.5 \%)$ of their husbands were farmers or daily labourers, and the rest either ran small businesses or were in office jobs. Majority $(56 \cdot 5 \%)$ of the subjects came from small-sized families consisting of $\leq 4$ members. About $10 \%$ of the women came from families with per-capita monthly income below the rural poverty line ${ }^{(21)}$ (Taka $659 /$ month). About $38 \%$ of the women were in their second trimester and the rest were in their third trimester.

The mean (SD) MUAC was $23 \cdot 7$ (SD 2.3) $\mathrm{cm}$ (Table 2). Prevalence of undernutrition (MUAC $<22.5 \mathrm{~cm}$ ) was $31.5 \%$ (Table 3). The distribution of dietary vitamin A intake was skewed towards the higher values and the median value was $732.5 \mathrm{RAE} / \mathrm{d}$ (Table 2 ). About $25 \%$ of the vitamin A intake came from animal foods and $75 \%$ from vegetable and fruits. The median intake of vitamin A from plant sources was $515 \mathrm{RAE} / \mathrm{d}$, while it was only $112 \mathrm{RAE} / \mathrm{d}$ from animal sources (Table 2). For more than half (53\%) of the subjects, the vitamin A intake was lower than the Recommended Dietary Allowance (RDA) $(<770 \mathrm{RAE} / \mathrm{d}$, Table 3). The serum retinol level was normally distributed, and the mean serum retinol concentration was $1.09 \mu \mathrm{mol} / \mathrm{l}$ (Table 2). Of the participants, $51 \%$ had low serum retinol concentration $(<1.05 \mu \mathrm{mol} / \mathrm{l})$, with $18.5 \%$ having $\mathrm{VAD}$ (serum retinol $<0 \cdot 70 \mu \mathrm{mol} / \mathrm{l}$ ) (Table 3 ).

The women in their third trimester of pregnancy had significantly $(P=0 \cdot 0001)$ lower serum retinol concentration than the women in their second trimester (Table 4). The women with MUAC $<22 \cdot 5 \mathrm{~cm}$ also had significantly

Table 2 Mid-upper arm circumference (MUAC), vitamin A intake and serum retinol concentration among pregnant women in Kapasia sub-district of Bangladesh

\begin{tabular}{|c|c|c|c|c|c|}
\hline Variable & $n$ & Mean & SD & Median & 25th-75th percentile \\
\hline \multicolumn{6}{|l|}{ Anthropometry } \\
\hline MUAC $(\mathrm{cm})$ & 200 & $23 \cdot 7$ & $2 \cdot 3$ & & \\
\hline \multicolumn{6}{|l|}{ Dietary vitamin A intake (RAE/d) } \\
\hline Animal source & 200 & & & $112 \cdot 5$ & $22 \cdot 5-237 \cdot 5$ \\
\hline Plant source* & 200 & & & $515 \cdot 0$ & $160 \cdot 0-867 \cdot 5$ \\
\hline Total & 200 & & & $732 \cdot 5$ & $331 \cdot 9-1068 \cdot 8$ \\
\hline \multicolumn{6}{|l|}{ Biochemistry } \\
\hline Serum retinol (vitamin A) $(\mu \mathrm{mol} / \mathrm{l}) \dagger$ & 184 & 1.09 & 0.42 & & \\
\hline
\end{tabular}

RAE, retinol activity equivalent.

For animal food, $1 \mathrm{RAE}=1 \mu \mathrm{g}$ of retinol; for plant food, $1 \mathrm{RAE}=12 \mu \mathrm{g}$ of $\beta$-carotene.

*Vegetables and fruits together.

tSixteen samples were lost during processing or haemolysed. 
Table 3 Prevalence of undernutrition, low vitamin A intake and vitamin A deficiency and night blindness among pregnant women in Kapasia sub-district of Bangladesh

\begin{tabular}{lrrrr}
\hline & \multicolumn{4}{c}{ Prevalence } \\
\cline { 3 - 5 } Variable & $N$ & $n$ & Percentage & $95 \% \mathrm{Cl}$ \\
\hline Undernutrition & 200 & 63 & $31 \cdot 5$ & $25 \cdot 5,38 \cdot 2$ \\
Vitamin A intake less than RDAt & 200 & 107 & $53 \cdot 5$ & $46 \cdot 6,60 \cdot 3$ \\
Vitamin A deficiency & 70 & 4 & $5 \cdot 7$ & $2 \cdot 2,13 \cdot 8$ \\
$\quad$ Second trimester & 114 & 30 & $26 \cdot 3$ & $19 \cdot 1,35 \cdot 1$ \\
$\quad$ Third trimester & 184 & 34 & $18 \cdot 5$ & $13 \cdot 5,24 \cdot 7$ \\
$\quad$ Total & & & & \\
Low vitamin A status & 70 & 21 & $11 \cdot 4$ & $7 \cdot 6,16 \cdot 8$ \\
$\quad$ Second trimester & 114 & 39 & $34 \cdot 2$ & $26 \cdot 1,43 \cdot 3$ \\
$\quad$ Third trimester & 184 & 60 & $32 \cdot 6$ & $26 \cdot 3,39 \cdot 7$ \\
$\quad$ Total & 200 & 4 & $2 \cdot 0$ & $0 \cdot 3,3 \cdot 6$ \\
Night blindness & & &
\end{tabular}

$N$, total number of subjects; $n$, number of subject with low status; RDA Recommended Dietary Allowance.

${ }^{*}$ Mid-upper arm circumference $<22.5 \mathrm{~cm}$

$\mathrm{t}<770 \mathrm{RAE} / \mathrm{d}$ (RAE, retinol activity equivalent).

$\ddagger$ Serum retinol $<0.70 \mu \mathrm{mol} / \mathrm{l}$.

\$Serum retinol between 0.70 and $<1.05 \mu \mathrm{mol} / \mathrm{l}$.

Table 4 Relationship between serum retinol concentration and gestational age, mid-upper arm circumference (MUAC) and dietary vitamin A intake among pregnant women in Kapasia sub-district of Bangladesh

\begin{tabular}{lrrrr}
\hline Variable & $n$ & Mean & $95 \% \mathrm{Cl}$ & $P$ value \\
\hline Gestational age (weeks) & & & & \\
$\quad$ 13-24 (second trimester) & 72 & 1.27 & $1.18,1.37$ & \\
$\quad>25$ (third trimester) & 112 & 0.98 & $0.91,1.06$ & 0.0001 \\
MUAC (cm) & 59 & 0.99 & $0.89,1.08$ & \\
$\quad<22.5$ & 125 & 1.14 & $1.07,1.22$ & 0.017 \\
$\quad \geq 22.5$ & 127 & 1.12 & $1.04,1.20$ & \\
$\quad \begin{array}{l}\text { Vitamin A intake (RAE/d) } \\
\quad<770^{*}\end{array}$ & 57 & 1.07 & $0.97,1.16$ & 0.42 \\
$\quad \geq 770$ & & & & \\
\hline
\end{tabular}

RAE, retinol activity equivalent

${ }^{*}$ Recommended Dietary Allowance $=770 \mathrm{RAE} / \mathrm{d}$.

$(P=0 \cdot 017)$ lower serum retinol concentration than the women with MUAC $\geq 22 \cdot 5 \mathrm{~cm}$. No relationship was found between dietary vitamin A intake and serum retinol concentration of the pregnant women.

There were statistically significant positive associations between serum retinol concentration and age at first marriage $(r=0 \cdot 14 ; P=0 \cdot 05), \operatorname{MUAC}(r=0 \cdot 235 ; P=0 \cdot 001)$, per-capita income $(r=0.155 ; \quad P=0.03)$, per-capita expenditure on food $(r=0.21 ; P=0.003)$ and wealth index $(r=0 \cdot 167 ; P=0 \cdot 023)$ of the women. On the other hand, there was a significant negative association between serum retinol and gestational age $(r=-0 \cdot 335$; $P=0 \cdot 0001)$. Factors influencing the serum retinol concentration were explored in more detail using backward stepwise multiple regression analysis (Table 5). When age at first marriage, gestational age, MUAC, dietary vitamin A intake, per-capita income, per-capita expenditure on food, owner of cultivation land and wealth index were included in the analysis and using a $P$ value of $0 \cdot 10$ for exclusion, only gestational age, MUAC, per-capita expenditure on food and wealth index were found to be
Table 5 Multiple regression analysis* for serum retinol concentration among pregnant women in Kapasia sub-district of Bangladesh

\begin{tabular}{lcccl}
\hline Variable & $B$ & SE $B$ & $\beta$ & $P$ value \\
\hline Gestational age (week) & -0.02 & 0.004 & -0.32 & 0.0001 \\
MUAC (cm) & 0.04 & 0.013 & 0.235 & 0.002 \\
Per-capita expenditure & 0.001 & 0.000 & 0.174 & 0.02 \\
$\quad$ on foodt & 0.048 & 0.023 & 0.159 & 0.04 \\
Wealth index & & \\
\hline
\end{tabular}

$B$, unstandardised coefficient; SE $B$, standard error of $B ; \beta$, standardised coefficient; MAUC, mid-upper arm circumference.

*Multiple $R=0.445 ; R^{2}=0.198 ;$ Adjusted $R^{2}=0.179 ; F$-ratio = 10.3 (df 4); $P=0.0001$.

†Taka/week

significantly independently related to the serum retinol concentration of these women, while the gestational age of the pregnant women bore a stronger but negative relationship with serum retinol concentration compared to other variables judged by comparable $\beta$ coefficients. The overall $F$-ratio was $10 \cdot 3(\mathrm{df}=4)$ and was highly significant $(P=0 \cdot 0001)$. The adjusted $R^{2}$ was 0.179 (multiple $R=0 \cdot 445$ ), suggesting that the variables in the equation accounted for $18 \%$ of the variance in serum retinol concentration.

\section{Discussion}

The present study reveals that there is a high prevalence of low vitamin A status in this group of rural Bangladeshi pregnant women and an association of serum retinol concentration with gestational age, undernutrition, expenditure on food and household wealth index of these women. The lack of association between serum retinol and vitamin A intake is somewhat unexpected, because a significant correlation has been reported in other studies ${ }^{(12,22)}$. One possible explanation is that in this study, the $24 \mathrm{~h}$ recall VASQ method was used to estimate the dietary vitamin A intake, which in this population may have been inadequate to capture their usual intake.

VAD has been defined as a public health problem when the prevalence of VAD, judged by serum retinol $<0.070 \mu \mathrm{mol} / \mathrm{l}$, among pregnant women is $>15 \%{ }^{(23)}$. In the present study, the prevalence of VAD was $18.5 \%$, thus indicating a significant public health problem among rural Bangladeshi pregnant women. The prevalence of low vitamin A status $(<1.05 \mu \mathrm{mol} / \mathrm{l})$ in the present study appears to be similar to that observed in the national survey in rural Bangladesh in $1997-98^{(12)}$; however, the prevalence of $\mathrm{VAD}$ is more evident in the national survey $(23.7 \%)$. In our study, $5 \cdot 7 \%$ of the women who were in the second trimester (13-24 weeks) had VAD. A study on pregnant women, between 20 and 30 weeks of gestation, in poor urban area of Bangladesh found $8.6 \%$ with $\mathrm{VAD}^{(24)}$, indicating that the pregnant women in the poor urban area may be more vulnerable than those in the 
rural area. However, it is worth mentioning that serum retinol concentration changes with the progress of pregnancy and thus it may be difficult to compare our study finding with other studies and to draw any conclusion.

Further, although almost all pregnant women who attended the CNP centres in the four unions of Kapasia sub-district during the survey period participated in the study, the study area has been covered by the nutritional intervention programmes including food supplements. This may have contributed to the lower prevalence of VAD in this population. Therefore, the results of the present study may not be representative of the wider rural population. Nevertheless, it is likely that for most pregnant women in this population it is no better, on the whole, than reported here.

It is important to note that sub-clinical infection or inflammation has been found to be associated with the reduction of serum retinol level ${ }^{(25)}$. Although we do not have any information on sub-clinical infection in the present study, at the time of blood collection all participants were apparently healthy.

A history of night blindness, an earliest clinical symptom of VAD, during the current pregnancy was also examined through the questionnaires. However, night blindness is a rare occurrence that requires large populations to obtain prevalence data ${ }^{(26)}$. In the present study, the sample size was too small to draw a conclusion about vitamin A status using a history of night blindness. Nevertheless, the existence of VAD as a public health problem could be inferred, considering that $2 \%$ of the pregnant women reported having night blindness during the current pregnancy. Studies have shown that night blindness from VAD is common among pregnant women, particularly in the last half of pregnancy ${ }^{(4)}$.

In the present study, we measured the dietary intake of the participants using the $24 \mathrm{~h}$ recall VASQ method and observed that nearly half of the women in the present study met the RDA for vitamin A intake. The nutritional survey in 1997-98 by $\mathrm{HKI}^{(12)}$ found that only $13 \%$ of pregnant women consumed at the level of RDA, where they used higher cut-off for RDA ( $<1000 \mathrm{RAE} / \mathrm{d})$.

We also explored the relationship between vitamin A status and various factors to identify the possible risk factors of $\mathrm{VAD}$ in this population. Bivariate analyses revealed that the women in the third trimester of pregnancy, despite the significantly $(P=0 \cdot 04)$ higher dietary intake of vitamin A (data not shown), had significantly $(P=0 \cdot 001)$ lower mean serum retinol level than those in the second trimester of pregnancy. In addition, the prevalence of VAD was almost five times higher among the women in the third trimester than those in the second trimester. Studies have shown that night blindness, a clinical symptom of VAD, is common among poor Nepalese pregnant women, especially during the last half of pregnancy ${ }^{(2,6)}$. Another study examining the plasma retinol at various stages of pregnancy showed that the mean value at the third trimester was significantly lower than that at the first and second trimesters ${ }^{(27)}$.

In the present study, malnourished (MUAC $<22.5 \mathrm{~cm}$ ) pregnant women were found to have statistically significantly lower serum retinol level than well-nourished (MUAC $\geq 22.5 \mathrm{~cm}$ ) pregnant women. Our finding is consistent with many studies. A study in Nepal showed that pregnant women who were night blind were more malnourished than pregnant women without night blindness, as judged by all anthropometric indices ${ }^{(6)}$. Another study on children in Bangladesh showed that low MUAC was associated with a low intake of vitamin A-containing foods as well as with low serum retinol levels ${ }^{(28)}$.

In the present study, there was no difference in the serum retinol level between the women who satisfied the RDA of vitamin A ( $\geq 770 \mathrm{RAE} / \mathrm{d})$ and those who did not satisfy the RDA of vitamin A $(<770 \mathrm{RAE} / \mathrm{d})$. Although the prevalence of VAD was almost three times higher among the women who did not satisfy the RDA compared to those who satisfied the RDA (data not shown), the difference was not statistically significant. As mentioned earlier, the $24 \mathrm{~h}$ recall VASQ method might have failed to capture the usual intake in this population and thus failed to show any association. On the contrary, de Pee et al. ${ }^{(22)}$ demonstrated that the $24 \mathrm{~h}$ recall VASQ method could produce data on vitamin A intake that was related to serum vitamin A level in a dose-dependent manner.

It might be possible that using bivariate analysis, the serum retinol levels of these women were confounded by the effect of various related factors. Therefore, we also carried out multiple regression analysis to identify factors that are independently related to the serum retinol level. The result indicates that serum retinol concentrations in rural Bangladeshi pregnant women were significantly influenced by gestational age, MUAC, per-capita expenditure on food and household wealth index. Furthermore, the results show that gestational age of the pregnant women bore a stronger but a negative relationship with serum vitamin A level. The wealth index and expenditure on food are indicators of socio-economic status. Earlier studies in Bangladesh also showed that the levels of serum retinol were lower in individuals from poorer socio-economic background than those from better circumstances $^{(12,24)}$.

In conclusion, the present study shows that VAD among rural pregnant women in Bangladesh is a significant public health problem. The pregnant women, especially in the third trimester, are more at risk of developing VAD. Poor nutritional status during pregnancy is also an important factor influencing their vitamin A status. Further, the data showed a trend of association between various socio-economic and vitamin A status of the pregnant women. Considering the possible implications of VAD during pregnancy, appropriate intervention should be implemented to improve the vitamin A status of these women. 


\section{Acknowledgements}

Conflicts of interest: None of the authors have any conflict of interest in relation to this study's findings.

Funding source: This research was supported by the internal funding of the School of Population Health, University of Queensland, Australia as part of $\mathrm{MPH}$ degree (V.L. and S.W.).

Author contributions: V.L. and S.W. were research students who collected all data, C.P.B. carried out biochemical analaysis, N.A. supervised dietary data collection, F.A., T.A. and S.A. together designed the project and supervised the overall progress. All authors contributed in writing.

Acknowledgements: We express our sincere thanks to the participants of this study. We acknowledge the technical support of the Helen Keller International, Dhaka, Bangladesh, for helping with dietary data collection. We thank BRAC, Dhaka, for allowing us to work in their CNP centres for sample collection.

\section{References}

1. World Health Organization (2003) Micronutrient deficiencies: combating vitamin A deficiency. http://www.who.int/ nut/vad.htm (accessed October 2005).

2. Katz J, Khatry SK, West KP Jr, Humphrey JH, LeClerq SC, Kimbrough E, Pohkrel PR \& Sommer A (1995) Night blindness is prevalent during pregnancy and lactation in rural Nepal. J Nutr 125, 2122-2127.

3. Christian P, Schulze K, Stoltzfus RJ \& West KP Jr (1998) Hyporetinolemia, illness symptoms, and acute phase protein response in pregnant women with and without night blindness. Am J Clin Nutr 67, 1237-1243.

4. Christian P (2002) Recommendations for indicators: night blindness during pregnancy - a simple tool to assess vitamin A deficiency in a population. $J$ Nutr 132, 2884S-2888S.

5. Suharno D, West CE, Muhilal, Karyadi D \& Hautvast JG (1993) Supplementation with vitamin A and iron for nutritional anaemia in pregnant women in West Java, Indonesia. Lancet 342, 1325-1328.

6. Christian P, West KP Jr, Khatry SK, Katz J, Shrestha SR, Pradhan EK, LeClerq SC \& Pokhrel RP (1998) Night blindness of pregnancy in rural Nepal - nutritional and health risks. Int J Epidemiol 27, 231-237.

7. West KP Jr, Katz J, Khatry SK et al. (1999) Double blind, cluster randomised trial of low dose supplementation with vitamin A or beta carotene on mortality related to pregnancy in Nepal. The NNIPS-2 Study Group. Br Med J 318, 570-575.

8. Christian P, West KP Jr, Khatry SK, LeClerq SC, KimbroughPradhan E, Katz J \& Shrestha SR (2001) Maternal night blindness increases risk of mortality in the first 6 months of life among infants in Nepal. J Nutr 131, 1510-1512.

9. Nandi B \& Tontisirin K (1995) A food-based approach to control vitamin A deficiency. In Empowering Vitamin A Foods: A Food-based Process for the Asia and Pacific Region, pp. 1-5 [Emorn Wasantwisut and GA Attig, editors]. Bangkok, Thailand/Salaya, Thailand/Bogor, Indonesia:
FAO Regional Office for Asia and the Pacific/Mahidol University Institute of Nutrition/South and East Asia Nutrition Research-cum-Action Network.

10. Olson J (1996) Chapter 11: Vitamin A. In Present Knowledge in Nutrition. Washington DC: ILSI.

11. Helen Keller International/Institute of Public Health Nutrition (1985) Bangladesh Nutritional Blindness Study, 1982-8. Dhaka, Bangladesh: HKI/IPHN.

12. Helen Keller International/Institute of Public Health Nutrition (1999) Vitamin A status throughout the lifecycle in rural Bangladesh: National Vitamin A survey 1997-98. Dhaka, Bangladesh: HKI/IPHN.

13. Bangladesh Rural Advancement Committee (2006) Kapasia Profile. Kapasia, Bangladesh: BRAC.

14. Rutstein S \& Johnson K (2004) The DHS Wealth Index. DHS Comparative Reports no. 6. Calverton, MD: ORC Macro.

15. De Pee S, Bloem MW, Halati S et al. (1999) 24-VASQ method for estimating vitamin A intake: reproducibility and relationship with vitamin A status. In Report of the XIX International Vitamin A Consultative Group Meeting, p. 96. Washington, DC: International Life Sciences Research Foundation.

16. De Pee S, Tjiong R \& Bloem M (2006) 24-VASQ Method for Estimating VA Intake. Indonesia: HKI Asia-Pacific Regional Office.

17. Helen Keller International (1988) Table of Nutrient Composition of Bangladeshi Foods: English Version with Particular Emphasis on Vitamin A Content, pp. 1-54. Dhaka, Bangladesh: HKI.

18. World Health Organization (1995) Pregnant and lactating women. In Physical Status: The Use and Interpretation of Anthropometry. WHO Technical Report Series no. 854, pp. 37-120. Geneva: WHO.

19. Bieri JG, Tolliver TJ \& Catignani GL (1979) Simultaneous determination of alpha-tocopherol and retinol in plasma or red cells by high pressure liquid chromatography. $A m \mathrm{~J}$ Clin Nutr 32, 2143-2149.

20. Ahmed F, Hasan N \& Kabir Y (1997) Vitamin A deficiency among adolescent female garment factory workers in Bangladesh. Eur J Clin Nutr 51, 698-702.

21. Bangladesh Bureau of Statistics (2003) Report of the Household Income and Expenditure Survey 2000. Dhaka, Bangladesh: BBS.

22. De Pee S, Bloem MW, Gorstein J, Sari M, Satoto, Yip R, Shrimpton R \& Muhilal (1998) Reappraisal of the role of vegetables in the vitamin A status of mothers in Central Java, Indonesia. Am J Clin Nutr 68, 1068-1074.

23. International Vitamin A Consultative Group (2001) 25 year progress in controlling vitamin A deficiency: looking to the future. In XX IVACG Meeting Program, p. 12. Washington, DC: ILSI.

24. Ahmed F, Mahmuda I, Sattar A \& Akhtaruzzaman M (2003) Anaemia and vitamin A deficiency in poor urban pregnant women of Bangladesh. Asia Pac J Clin Nutr 12, 460-466.

25. Filteau SM \& Tomkins AM (1994) Micronutrients and tropical infections. Trans $R$ Soc Trop Med Hyg 88, 1-3.

26. World Health Organization (1996) Indicators for Assessing Vitamin A Deficiency and Their Application in Monitoring and Evaluating Intervention Programmes. WHO/NUT/ 96.10. Geneva: WHO

27. Bodansky O, Lewis JM \& Lillienfeld MC (1943) The concentration of vitamin A in the blood plasma during pregnancy. J Clin Invest 22, 643-647.

28. Hussain A, Lindtjorn B \& Kvale G (1996) Protein energy malnutrition, vitamin A deficiency and night blindness in Bangladeshi children. Ann Trop Paediatr 16, 319-325. 